\title{
Universal Size-Shape Effect Law Based on Comprehensive Concrete Fracture Tests
}

\author{
Christian G. Hoover ${ }^{1}$ and Zdeněk P. Bažant, Hon.M.ASCE ${ }^{2}$
}

\begin{abstract}
The universal size-shape effect law is a law that describes the dependence of nominal strength of specimen or structure on both its size and the crack (or notch) length, over the entire range of interest, and exhibits the correct small-size and large-size asymptotic properties as required by the cohesive crack model (or crack band model). The main difficulty has been the transition of crack length from 0 , in which case the size effect is Type 1, to deep cracks (or notches), in which case the size effect is Type 2 and is fundamentally different from Type 1, with different asymptotes. In this transition, the problem is not linearizable because the notch is not much larger than the fracture process zone. The previously proposed universal law could not be verified experimentally for the Type 1-Type 2 transition because sufficient test data were lacking. The current study is based on recently obtained comprehensive fracture test data for three-point bend beams cast from one batch of the same concrete and cured and tested under identical conditions. The test data reveal that the Type 1-Type 2 transition in the previous universal law has insufficient accuracy and cannot be captured by Taylor series expansion of the energy release rate function of linear elastic fracture mechanics. Instead, the size effect for a zero notch and for the transitional range is now characterized in terms of the strain gradient at the specimen surface, which is the main variable determining the degree of stress redistribution by the boundary layer of cracking. The new universal law is shown to fit the comprehensive data quite well, with a coefficient of variation of only 2.3\%. DOI: 10.1061/(ASCE)EM.1943-7889.0000627. @ 2014 American Society of Civil Engineers.
\end{abstract}

Author keywords: Quasi-brittle fracture; Cohesive crack; Scaling; Asymptotics of fracture; Failure of structures; Statistics of experimental data.

\section{Introduction}

Progress in the modeling of concrete fracture and introduction of fracture concepts into design codes and practice has been impeded by the unavailability of a comprehensive database for fracture alone. The literature features a vast number of fracture data (BecqGiraudon 2000; Rocco 1995; Sabnis and Mirza 1979; Bažant and Becq-Giraudon 2002; Bažant and Planas 1998; Malvar and Warren 1988; Nallathambi 1986; Petersson 1981; Carpinteri et al. 1995; Bažant and Pfeiffer 1987; Tang et al. 1996; Karihaloo et al. 2003), but they all cover only rather limited ranges of specimen size, initial notch depth, and postpeak response and have been performed on different concretes, on different batches of supposedly the same concrete, at different ages, at different environmental conditions, at different rates, with different test procedures, and on specimens of different types and dimensions. Combining all these data produces a database with enormous scatter and makes the modeling highly ambiguous because the effect of these differences is understood much less than the fracture itself.

\footnotetext{
${ }^{1}$ Postdoctoral Research Associate, Dept. of Civil and Environmental Engineering, Massachusetts Institute of Technology, Cambridge, MA 02139; formerly, Graduate Research Assistant, Dept. of Civil and Environmental Engineering, Northwestern Univ., 2145 Sheridan Rd., CEE/A135, Evanston, IL 60208.

${ }^{2}$ McCormick Institute Professor and W. P. Murphy Professor of Civil and Mechanical Engineering and Materials Science, Northwestern Univ., 2145 Sheridan Rd., CEE/A135, Evanston, IL 60208 (corresponding author). E-mail: z-bazant@ northwestern.edu

Note. This manuscript was submitted on August 16, 2012; approved on March 14, 2013; published online on February 14, 2014. Discussion period open until August 1, 2014; separate discussions must be submitted for individual papers. This paper is part of the Journal of Engineering Mechanics, Vol. 140, No. 3, March 1, 2014. @ASCE, ISSN 0733-9399/ 2014/3-473-479/\$25.00.
}

To remedy this situation, the U.S. DOT provided funding to carry out comprehensive fracture tests on specimens made from the same batch of one typical modern concrete of the same age and the same curing conditions, using beam specimens of size range 1:12.5 and notch depths from 0 to $30 \%$ of cross section and including the entire postpeak softening. In total, 18 different beam geometries (families of beams) were cast. These data, reported and analyzed in two previous papers and a companion paper (Hoover et al. 2013; Hoover and Bažant 2013, 2014), will be used here to develop an improved analytical formula for the effects of size and crack or notch depth on the nominal strength of specimens.

Development of such a formula has been attempted before (Bažant and Yu 2004, 2009; Bažant 1995, 1997) based on the technique of asymptotic matching, but experimental verification was incomplete. The objective of this paper is to develop and experimentally verify a new formula for the combined effects of structure size and crack or notch depth, called the universal size-shape effect law (because the variation of crack depth represents an effect of structure shape, or geometry). One particular objective is to bridge the previously clarified size effects in specimens with no cracks or notches and in specimens with deep notches or cracks called the Type 1 and Type 2 size effects, respectively. These two size effects are fundamentally different and transition between them has been one important gap in experimental information. The problem is difficult because, in the transition, the crack or notch is not much larger than the fracture process zone (FPZ), which precludes linear approximations.

\section{Review of Size Effect and Crack Length Effect}

In elastoplasticity, the nominal strength of geometrically similar structures, defined as

$$
\sigma_{N}=c_{N} \frac{P_{u}}{b D}
$$


is independent of the structure size $D(P=$ maximum load; $b$ $=$ structure width; and $c_{N}=$ dimensionless constant chosen for convenience). The size effect is understood as any dependence of $\sigma_{N}$ on $D$, which is a feature typical of fracture or damage mechanics.

According to linear elastic fracture mechanics (LEFM), which applies to homogeneous perfectly brittle materials, and for geometrically similar structures with similar cracks, $\sigma_{N} \propto D^{-1 / 2}$, which is the strongest possible size effect. For quasi-brittle materials such as concrete, one can distinguish two simple types of size effect. Considering geometrically similar structures containing at maximum load cracks or notches that are sufficiently larger than the FPZ, one has the Type 2 size effect, which is well described by the size effect law (SEL)

$$
\sigma_{N}=\frac{B f_{t}^{\prime}}{\sqrt{1+D / D_{0}}} \quad(\text { Type } 2)
$$

Here $B$ and the transitional structure size $D_{0}$ are empirical parameters to be identified by data fitting and $f_{t}^{\prime}=$ tensile strength of concrete, introduced for convenience. Eq. (2) was derived (Bažant 1984) by simple energy release analysis and later by several other ways, especially by asymptotic matching based of the asymptotic power scaling laws for very large and very small $D$ (Bažant and Planas 1998; Bažant 2005). In the standard size effect plot of $\log \sigma_{N}$ versus $\log D$, Eq. (2) gives a smooth transition from a horizontal asymptote to an inclined asymptote of slope $-1 / 2$ (Fig. 1).

By means of asymptotic expansion of equivalent LEFM, it was further shown (Bažant et al. 1989; Bažant and Kazemi 1991) that

$$
B f_{t}^{\prime}=E^{\prime} G_{f} / g_{0}^{\prime} c_{f}, \quad D_{0}=c_{f} g_{0}^{\prime} / g_{0}
$$

where $g_{0}=g\left(\alpha_{0}\right) ; g_{0}^{\prime}=g^{\prime}\left(\alpha_{0}\right) ; \alpha=a / D=$ relative crack length; $\alpha_{0}=a_{0} / D=$ initial value of $\alpha ; g(\alpha)=k^{2}(\alpha)=$ dimensionless energy release rate function $g(\alpha)$ of LEFM; $k(\alpha)=b \sqrt{(D) K_{I} / P}$ where $K_{I}=$ stress intensity factor, $P=$ load; $g^{\prime}(\alpha)=\mathrm{d} g(\alpha) / \mathrm{d} \alpha$; $E^{\prime}=E=$ Young's modulus for plane stress and $E^{\prime}=E /\left(1-\nu^{2}\right)$ for plane strain (where $\nu=$ Poisson ratio); $G_{f}=$ initial fracture energy $=$ area under the initial tangent of the cohesive softening stress-separation curve; $c_{f}=$ characteristic length, which represents about a half of the FPZ length and may be expressed as $c_{f}=\gamma_{s} l_{0}$, where $\gamma_{s}=$ material-dependent coefficient, for the present specimens equal to 0.29 (Bažant and Yu 2011; Cusatis and Schauffert 2009); and $l_{0}=E G_{f} / f_{t}^{\prime 2}=$ Irwin's material characteristic length (Irwin 1958). $G_{f}, c_{f}$, and $l_{0}$ are all considered to be independent of structure size, i.e., as constants, because they are the characteristics of the cohesive crack softening law, which itself is a material property (note the difference of $G_{f}$ from the total fracture energy, which represents the total area under the cohesive softening
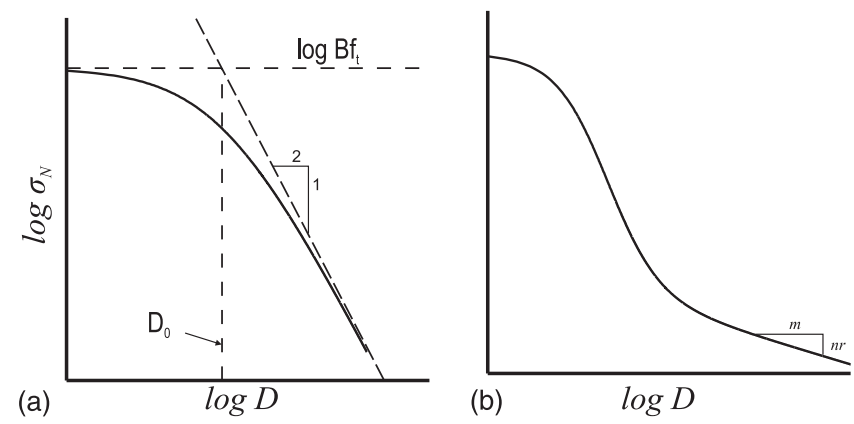

Fig. 1. Dependence of $\sigma_{N}$ on structure size $D$ of beams with (a) deep notches and (b) no notch stress-separation law, and the fact that $G_{f}$ is not equal to the energy release rate, which can vary with the size and distance from notch tip; on the other hand, $G_{F}$ is equal to the energy release rate in an infinitely large specimen).

Based on Taylor series expansion of function $g(\alpha)$, Eq. (2) may be rewritten as (Bažant and Kazemi 1991; Bažant et al. 1989)

$$
\sigma_{N}=\sqrt{\frac{E^{\prime} G_{f}}{g_{0} D+g_{0}^{\prime} c_{f}}} \quad(\text { Type } 2)
$$

Because function $g(\alpha)$ or $k(\alpha)$ embodies information on the effects of crack length and structure geometry (or shape), Eq. (4) is actually a size-shape effect law for Type 2 failures.

The Type 1 size effect is observed in structures of the so-called positive geometry (Bažant and Planas 1998), which fail under controlled load as soon as the macrocrack initiates from a smooth surface. In this case, $\sigma_{N}$ approaches, for large $D$, a constant value (a horizontal asymptote in the size effect plot), since the Weibull statistical size effect (Weibull 1939, 1951) is unimportant. For three-point bend beams, it is indeed unimportant. Why? - because the zone of high stress is rather concentrated, even in absence of a notch. This prevents the critical crack from forming at widely different locations of different random local strength (for the same reason, the statistical size effect is negligible in Type 2 failures also). The statistical size effect is also unimportant for small enough specimens of any geometry, for which the structure is not much larger than the FPZ. The deterministic size effect is also called energetic, because it can be derived from the limit case of energy release when the crack length approaches zero.

When, however, the zone of high stress in Type 1 failures occupies a large part of the structure, as in tensile loading of bars or in four-point bending, the statistical size effect is not negligible and for large sizes becomes important. Then the large size asymptote for Type 1 size effect is, in the log-log plot, a downward inclined straight line of a slope $-n / m$, which is much milder than the slope of $-1 / 2$ for LEFM (Weibull 1939, 1951) (Fig. 1); here $m$ $=$ Weibull modulus and $n=$ number of spatial dimensions of fracture scaling ( $n=2$ for the present tests). The small-size asymptote is also a horizontal line and, for medium sizes, the size effect is a transition between these two asymptotes. In absence of the statistical size effect

$$
\sigma_{N}=f_{r \infty}\left(1+\frac{r D_{b}}{\bar{D}+l_{p}}\right)^{1 / r} \quad \text { (Type } 1, \text { deterministic, or energetic) }
$$

and in presence of the statistical size effect

$$
\sigma_{N}=f_{r \infty}\left[\left(\frac{l_{s}}{l_{s}+D}\right)^{r n / m}+\frac{r D_{b}}{\bar{D}+l_{p}}\right]^{1 / r}
$$

(Type 1, energetic-statistical)

Here $f_{r \infty}, D_{b}, l_{p}, l_{s}$, and $r$ are empirical constants to be determined from tests; $f_{r \infty}=$ nominal strength for very large structures, assuming no statistical size effect (in the special case of very large beams, $f_{r \infty}$ represents the flexural strength, also called the modulus of rupture); and $D_{b}=$ depth of the boundary layer of cracking (roughly equal to the FPZ size). In all previous works, $\bar{D}=D=$ same characteristic structure size as used for the Type 2 size effect [Eq. (4)]. The reason for placing an overbar on $D$ is that $\bar{D}$ is later redefined in terms of the strain gradient.

Furthermore, $l_{s}=$ statistical characteristic length and $l_{p}$ $=$ material characteristic length, which is related to the maximum 
aggregate size $d_{a}$. Introducing $l_{p}$ is necessary for mathematical reasons, as a means to satisfy the asymptotic requirement of a finite plastic limit for $D \rightarrow 0$ while ensuring the effect of $l_{p}$ to be negligible for $D \gg l_{p}$. However, if the structure is larger than $10 l_{p}$, one can set $l_{p} \approx 0$, which corresponds to the original formulation of the Type 1 law. $l_{p}$ differs from Irwin's (1958) characteristic length $l_{0}$. Also in the limit of $m \rightarrow \infty$, the statistical Eq. (6) reduces to the deterministic Eq. (5). Further note that scaled tests of three-point bend beams with a notch of $\alpha_{0} \geq 0.15$ have been shown to yield virtually the same fracture parameters $G_{f}$ and $c_{f}$ as the tests with $\alpha_{0}=0.50$ (Tang et al. 1996), which is the reason why tests with $\alpha_{0}=0.50$ were deemed unnecessary for present comprehensive test program.

\section{Type 1 Size Effect in Terms of Boundary Strain Gradient}

An asymptotic expansion of LEFM function $g(\alpha)$ for initiating cracks and consideration of the limit $\alpha_{0} \rightarrow 0$ indicated that (Bažant 1997, 2005)

$$
D_{b}=-c_{f} g^{\prime \prime}(0) / 4 g^{\prime}(0), \quad f_{r \infty}=\sqrt{E^{\prime} G_{f} / c_{f} g^{\prime}(0)}
$$

where typically $g^{\prime \prime}(0)<0$. However, these expressions must be suspected of poorer accuracy because $g(0)=0$, and approximations by higher-order derivatives always have much larger errors. The comprehensive tests of Hoover et al. (2013) confirmed this suspicion. When the Type 2 SEL [Eq. (4)] was optimally fitted to the comprehensive test results (Hoover and Bažant 2013) for $\alpha_{0} \geq 0.15$, the value of $f_{r \infty}$ predicted by Eq. (7) using function $g(\alpha)$ is 1.33 times smaller than necessary to fit the no-notch test results and the value of $D_{b}$ is 2.1 times smaller. Thus, although the form of Eq. (5) obtained as the limit case of equivalent LEFM is correct, Eq. (7) gives parameter values that are too inaccurate and must therefore be abandoned as a consequence of the comprehensive test results of Hoover et al. (2013). It also means that Eq. (7) cannot be used to determine $G_{f}$, which means the only way to determine $G_{f}$ is to perform size effect tests on a large size range of beams with deep relative notches.

The Type 1 size effect law has originally been derived (Bažant and $\mathrm{Li}$ 1995) by considering the stress redistribution in beam bending caused by the finite depth $D_{b}$ of the boundary layer of cracking. This depth is roughly the same as the size of the FPZ of deep cracks and is about seven times $d_{a}$. The stress redistribution is essentially local and depends on how much the normal strain $\varepsilon_{n}$ at surface, in the direction parallel to surface, changes over the depth $D_{b}$ of the boundary layer of cracking. As suggested in Eq. 2.49 of Bažant (2005), this change may be characterized in terms of the strain gradient $\varepsilon_{, n}=\partial \varepsilon / \partial x_{n}$, where $x_{n}$ is the coordinate normal to the surface, oriented outward. The ratio $\varepsilon / \varepsilon_{, n}$ has the dimension of length and is proportional to the structure size $D$ when geometrically similar structures are considered. This suggests redefining $\bar{D}$ in Eqs. (5) and (6) as follows:

$$
\bar{D}=\frac{2 \varepsilon}{\psi \varepsilon, n}
$$

The reason for factor 2 is to make $\bar{D}$ coincide with the actual depth $D$ of a slender beam subjected to bending moment $M$ alone (to verify this fact, note that $\varepsilon$ at surface is equal to $\varepsilon_{, n} D / 2$, according to the plane cross section hypothesis). Factor $\psi(S / D)$ is introduced as a geometry factor, defined as 1 for a slender beam. The strain gradient should be obtained by elastic analysis with no crack and not by fracture analysis with a crack, because it represents the modification of elastic analysis needed to approximate the cohesive fracture effect.

The beams tested in Hoover et al. (2013) were not slender. They had a span-to-depth ratio $S / D=2.176$, and therefore $\psi(S / D) \neq 1$. According to the exact elastic solution (e.g., Timoshenko and Goodier 1951, p. 101), the stresses at the midspan cross section vary with transverse coordinate $y$ as follows:

$$
E \varepsilon(y)=\frac{3 P}{2 c^{3}}\left(\frac{S}{4}-\frac{c}{\pi}\right) y+\frac{P}{2 \pi c}+\frac{P}{\pi c}\left(\frac{y^{3}}{2 c^{3}}-\frac{3 y}{10 c}\right)
$$

Hence

$$
\frac{\varepsilon}{\varepsilon_{, n}}=\frac{\varepsilon(c)}{\varepsilon_{, y}(c)}=\frac{\psi}{2} D \quad \text { with } \psi=0.896
$$

where $c=D / 2$; and $S=$ span. Calculation of factor $\psi(S / D)$ for different $S / D$ makes it possible to infer the size effect for beams with $S / D$ other than 2.176 .

Using an elastic solution to determine factor $\psi$ might seem debatable. Wouldn't a solution with the cohesive crack model or a damage model be more appropriate? Not in the present context, because the purpose of $\varepsilon_{, n}$ is to introduce a correction of elasticity to approximate the solution with the cohesive crack or damage model, for which no correction for strain gradient is needed.

Another debatable point might be that the data fitted here are for specimens of the same width $b$ (which means using two-dimensional scaling). Wouldn't a proportional increase of $b$ (i.e., threedimensional scaling) be more appropriate? Not really, because it would bring about additional problems. Near the side walls there is a layer of constant thickness in which the crack must be nonplanar and the crack front curved (which is called the shear-lip phenomenon). Thus, if $b$ were varied, then different portions of the specimen thickness $b$ would be occupied by the surface layer. This is a small effect but it could cause the average fracture energy over width $b$ to vary (slightly) and would create additional scatter in the size effect trends.

\section{Universal SEL}

When the crack at failure is neither negligible nor large, the size effect trend is expected to be some sort of a transition between the Types 1 and 2. To clarify this transition and thus obtain a combined, or universal, size-shape effect law has been one key objective of the comprehensive fracture tests (Hoover et al. 2013).

In absence of comprehensive test data, there have been two previous purely theoretical attempts at a universal size effect that would be bridging Type 1 and Type 2 size effects. The first attempt (Bažant 1996; Bažant and Li 1996) led to a formula in which the transition was not smooth. A smooth formula was derived in Bažant and $\mathrm{Yu}$ (2009), but it could not be checked against experiments. The comprehensive fracture tests now make possible such a check, and show that the previous attempts to bridge the Type 1 and 2 size effects were not accurate enough.

An improved universal SEL (USEL) with the strain gradient could be derived by a similar asymptotic matching procedure as in Bažant and $\mathrm{Yu}$ (2009). However, it will be shorter to present first this universal law and then demonstrate that is has the correct asymptotic properties. Considering also the statistical size effect, the nonstatistical and statistical USELs may be stated as follows: 


$$
\sigma_{N}=\left[\frac{E^{\prime} G_{f}}{g_{0} D+(1-\lambda) c_{f} g_{0}^{\prime}+\lambda E^{\prime} G_{f} / f_{r \infty}^{2}}\right]^{1 / 2}\left(1+\frac{r \lambda D_{b}}{\bar{D}+l_{p}}\right)^{1 / r}
$$

$$
\begin{aligned}
\sigma_{N}= & {\left[\frac{E^{\prime} G_{f}}{g_{0} D+(1-\lambda) c_{f} g_{0}^{\prime}+\lambda E^{\prime} G_{f} / f_{r^{\infty}}^{2}}\right]^{1 / 2} } \\
& \times\left[\left(\frac{\lambda l_{s}}{D+l_{s}}\right)^{r n / m}+\frac{r \lambda D_{b}}{\bar{D}+l_{p}}\right]^{1 / r}
\end{aligned}
$$

in which an empirical Type 1 to 2 transition parameter is introduced and defined as

$$
\lambda=e^{-\left[\alpha_{0}^{k}\left(\bar{D} / d_{a}\right)^{p} / q\right]}
$$

Here $\bar{D}$ is given by Eq. (8); and $G_{f}, c_{f}, f_{r \circ}, D_{b}, r, k, p, q, l_{p}$, and $l_{s}$ are parameters to be calibrated by data fitting, whereas $\psi$ is calculated from the strain profile. The only purpose of the transition parameter $\lambda$ is to provide a smooth transition between the Type 1 and 2 behaviors. Thus, it is justified as an asymptotic matching parameter, with asymptotic cases $\lambda=1$ for no-notch specimens and $\lambda=0$ for deep notch specimens. Other smooth expressions for $\lambda$ were also tried (e.g., $\lambda=e^{-\left(\alpha_{0} / k\right)^{p}}$ and $\lambda=e^{-\left(\alpha_{0} D / k d_{a}\right)^{p}}$ ) but did not fit the data as well. The surface $\lambda(\alpha, D)$ at the location of each beam size used in Hoover et al. (2013) and overall is pictured in Fig. 2.

According to the method of asymptotic matching, which is the only way to formulate the SEL analytically, Eqs. (11) and (12) are justified by checking that they match the following two basic asymptotic trends:

1. For no notch, on setting $\alpha=0, g_{0}=0$, and $\lambda=1$ and using $\psi=0.896$, Eqs. (11) and (12) reduce to the deterministic or statistical Type 1 SELS in Eqs. (5) and (6); and

2. For deep notches, on setting $\lambda=0$, both Eqs. (11) and (12) reduce to the Type 2 size effect law in Eq. (2).

Previously (Bažant and Yu 2009), it was further shown that the Type 1 and 2 SELS satisfy the large-size and small-size asymptotic properties of the cohesive crack model applied to Type 1 and 2 failures.

Furthermore, it was experimentally confirmed that, within the range of inevitable experimental scatter, the SEL of Type 2 gives about the same values of fracture energy $G_{f}$ when applied to notched fracture specimens of different geometries [e.g., notched beam bending, edge-notched tension, eccentric compression (Bažant and Pfeiffer 1987)] [this check was made before function $g(\alpha)$, needed to also obtain $c_{f}$, was introduced into the SEL].

Eqs. (11)-(13) also give the correct prediction for an unnotched direct tension specimen. Indeed, in this case, $\varepsilon_{, n}=0, \bar{D} \rightarrow \infty, \lambda=1$, and $g_{0} \rightarrow 0$. Eq. (12) thus reduces to $\sigma_{N}=f_{r \infty}\left[l_{s} /\left(D+l_{s}\right)\right]^{m / n}$, which correctly gives, for large $D$, the pure Weibull statistical size effect $\sigma_{N}=f_{r \infty} D^{-m / n}$.

\section{Verification of USEL by Comprehensive Fracture Tests}

To calibrate the deterministic USEL, the mean of the data was computed separately for each family of identical specimens from the comprehensive fracture tests (Hoover et al. 2013) (these means are shown in the figures as the large and thin $x$ points). Then all these means were optimally fitted by Eq. (11). The trust-region-reflective optimization algorithm (Coleman and Li 1994, 1996) was used. It would not have been meaningful to fit all the unknown parameters concurrently because every one of them would then be influenced by all the data.

In particular, the fracture parameters $G_{f}$ and $c_{f}$ should not be influenced by the data for beams with no notches (Type 1 data) or shallow notches, and $f_{r \infty}, D_{b}, l_{p}$, and $r$ should not be influenced by the data for deep notches. Therefore, these parameters were determined first by separate fitting of specimens with deep notches $(\alpha=0.30,0.15)$ and specimens with shallow or no notches $(\alpha=0)$, and only the remaining ones, i.e., $k, p$, and $q$ were identified by simultaneous optimal fitting of all the data. Only the nonstatistical USEL in Eq. (11) was considered because parameters $m$ and $l_{s}$ for the Weibull statistical size effect cannot be identified from the present data. They are nevertheless well known from other studies (Bažant et al. 2007). Nonlinear fitting of the Type 1 SEL [Eq. (5)] to the notchless $(\alpha=0)$ beams gave (Hoover and Bažant 2013)

$$
D_{b}=73.2 \mathrm{~mm}, \quad l_{p}=126.6 \mathrm{~mm}, \quad f_{r, \infty}=5.27 \mathrm{MPa}, \quad r=1 / 2
$$

Actually, the optimization gave $r=0.52$, but replacing it with $r=0.5$ was found to have increase the COV of Type 1 data fits negligibly (from 1.907 to $1.909 \%$ ).

The size range 1:12.5 was large enough to identify all the fracture parameters in Eq. (5). $G_{f}$ and $c_{f}$ were identified by weighted nonlinear regression of the Type 2 SEL [Eq. (4)] run jointly for the
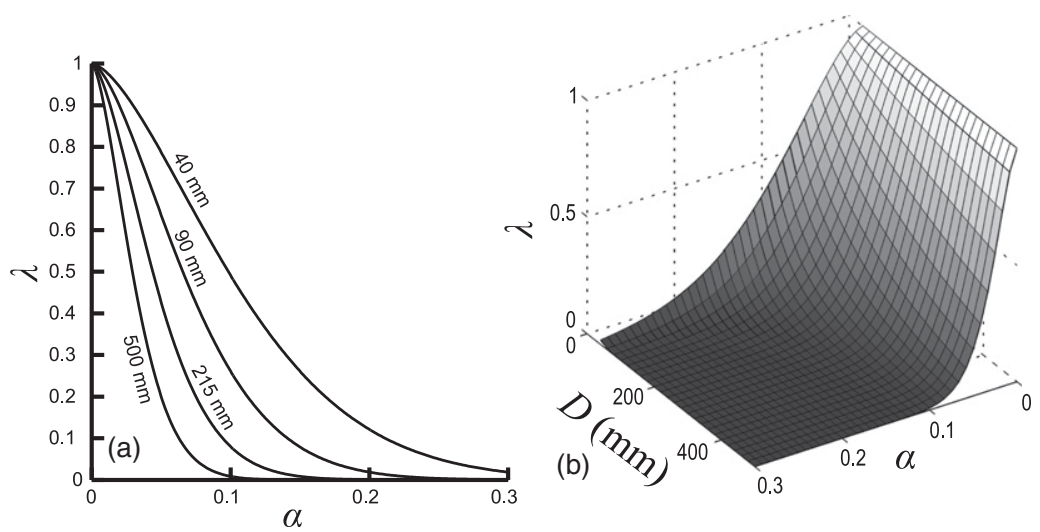

Fig. 2. Trend of the transition function $\lambda(\alpha, D)$ (a) at location of each beam size and (b) entire surface 
$\alpha=0.30$ and 0.15 beams, with different $g_{0}$ and $g_{0}^{\prime}$ for each $\alpha$. The means in each family were assigned the weights $w_{i}=\sigma_{i}^{-2}$ $/ \sum_{i=1}^{N} \sigma_{i}^{-2}$, representing the inverse variance of each beam family normalized with the sum of the inverse variances for all $N$ families $(N=8)$. The optimization gave

$$
G_{f}=56.25 \mathrm{~N} / \mathrm{m}, \quad c_{f}=29.79 \mathrm{~mm}
$$

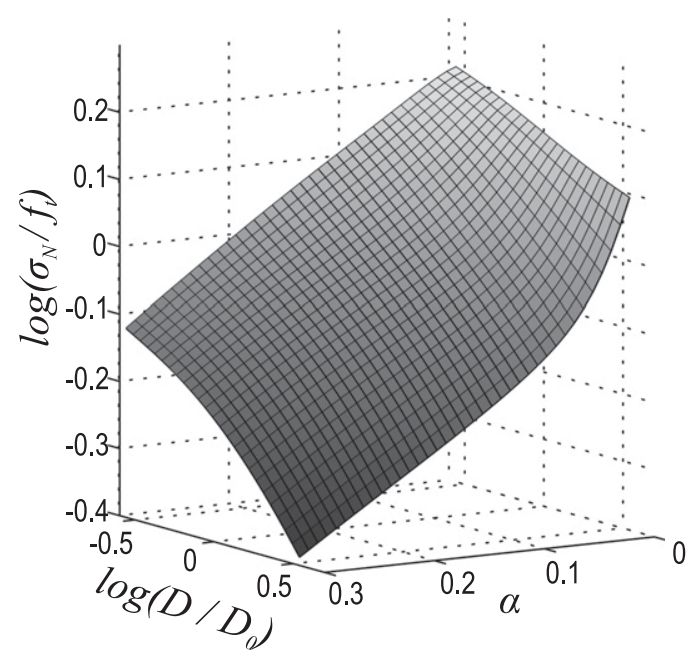

Fig. 3. Entire USEL surface; COV of fit is $2.3 \%$
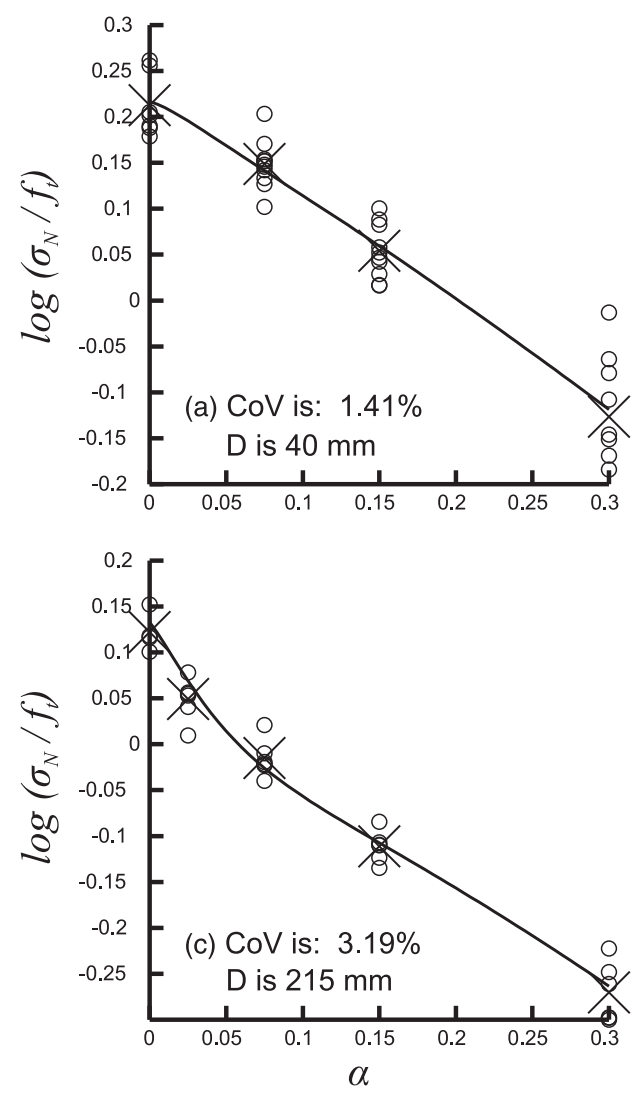

Fig. 4. Effect of relative crack length on the normalized nominal strength of data from Hoover et al. (2013) optimally fitted by the USEL [Eq. (11)]; the COVs of each fit are shown
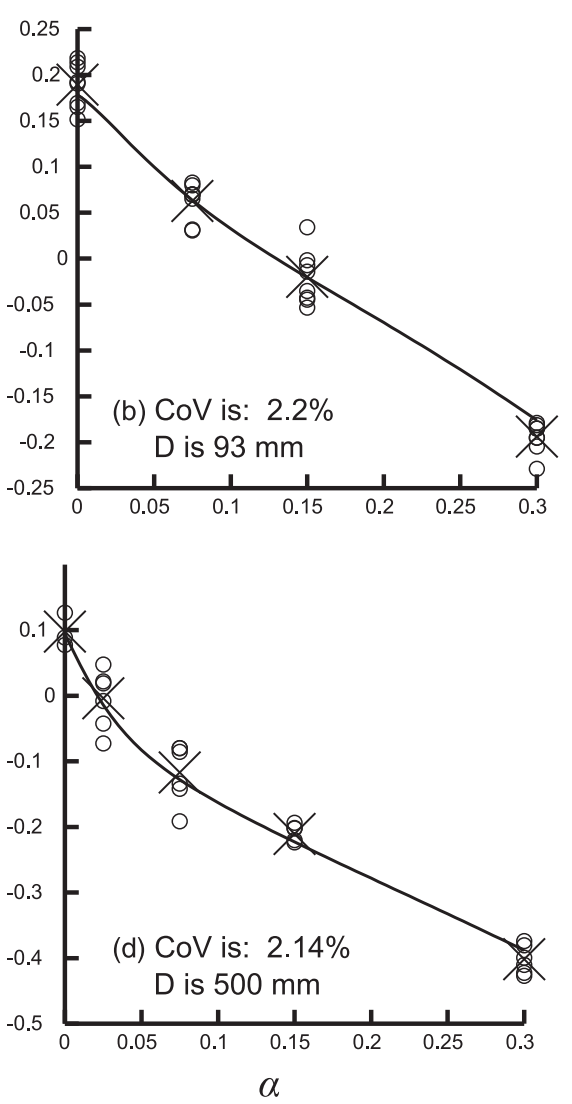

The optimization of the remaining parameters, all belonging to the transition function $\lambda(\alpha, D)$ [Eq. (13)], furnished $k=1.57$, $p=0.755$, and $q=0.108$.

The surface of the optimized USEL is pictured in Fig. 3, where the contour lines are the lines of constant $D$ or constant $\alpha$. The COV of fit of the entire surface (defined as the RMS error divided by the mean of all data) is only $2.30 \%$. For simplicity, the optimized parameters in the transition function may be rounded as

$$
k=3 / 2, \quad p=3 / 4, \quad q=1 / 9
$$

which makes the COV only slightly higher $(2.45 \%)$.

Although it seems that these parameter values can be kept the same even for other specimen geometries, recalibration may be needed for good accuracy.

The USEL can be drawn for a fixed $\alpha$, which gives a size effect plot of $\log \left(\sigma_{N} / f_{t}^{\prime}\right)$ versus $\log \left(D / D_{0}\right)$, or at a fixed relative depth $D / D_{0}$, which gives the plot of $\log \left(\sigma_{N} / f_{t}^{\prime}\right)$ versus $\log (\alpha)$. In Figs. 4 and 5, these plots are created and compared with the data from Hoover et al. (2013). The COV for each curve is indicated in the figures. It is seen that the curves fit the data satisfactorily. The largest $\mathrm{COV}$, equal to $3.8 \%$, comes from the size effect plot for $\alpha=0.025$, which is in the transition. The remaining COV values are less than $3.2 \%$, and four of them are less than $2 \%$.

\section{Remaining Questions}

It must be admitted that the experimental verification of the capability of the present universal size-shape effect law is not complete as 

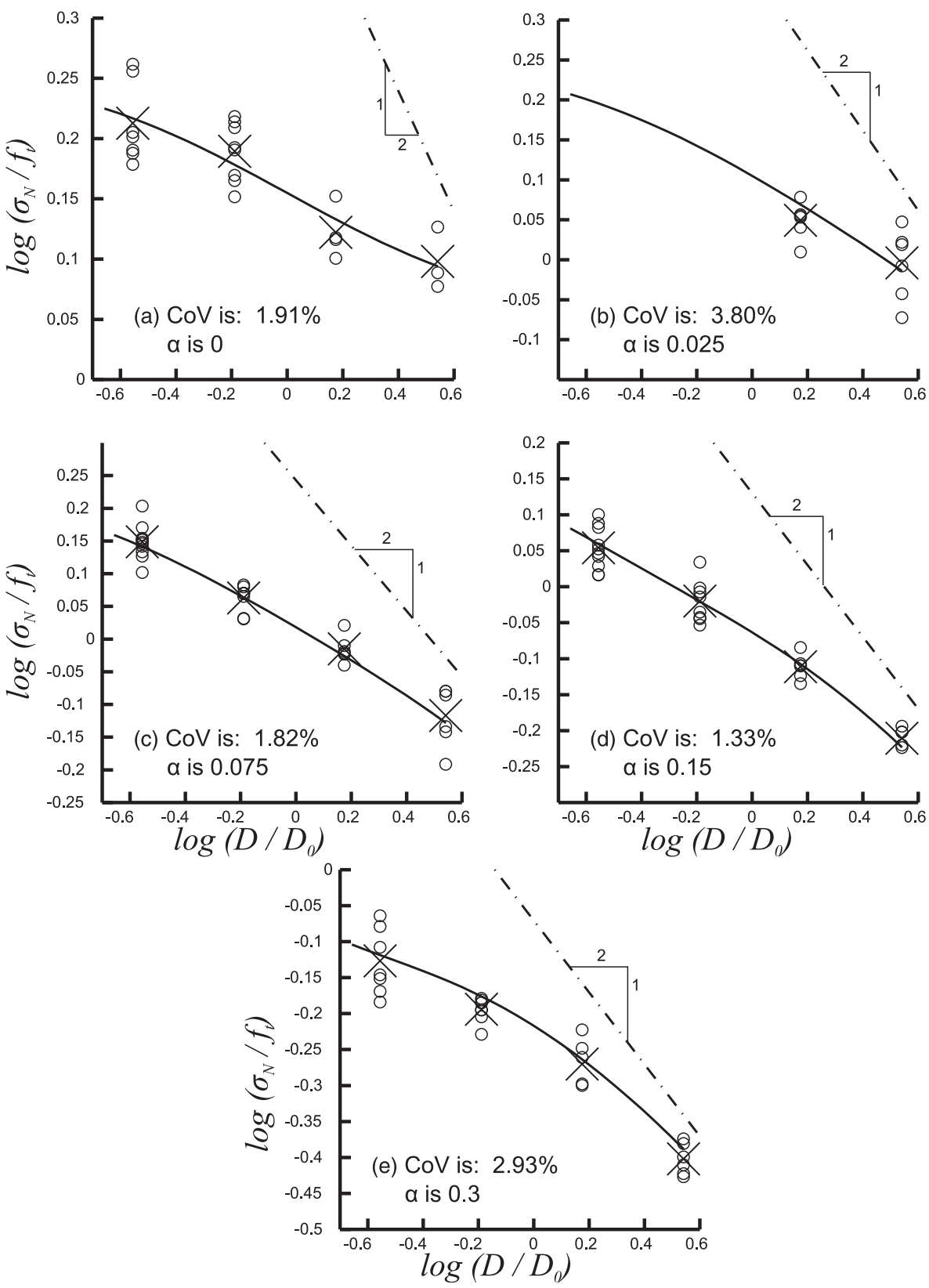

Fig. 5. Effect of normalized structure size on the normalized nominal strength of the data from Hoover et al. (2013) optimally fitted by the USEL [Eq. (11)]; the COVs of each fit are shown

far as the structure geometry (or shape) is concerned. That the Type 2 size effect law, with the same $G_{f}$, can fit the nominal strength values measured on different types of fracture specimens, including threepoint bend, double-edge-notched tensile, and edge-notched eccentric compression specimens, has been experimentally verified in Bažant and Pfeiffer (1987).

Because Eq. (8) describes a local effect, for which remote boundaries are irrelevant, this equation for $\bar{D}$ should, in theory, apply for any geometry. However, this remains to be proven experimentally. The transition function $\lambda$ is not completely local, and therefore its applicability to other geometries is less certain. The applicability of $D$ and $\lambda$ to other geometries could also be verified computationally, using the cohesive or crack-band model. Extending these function to a very different material would surely necessitate recalibration.

\section{Conclusions}

1. The use of the dimensionless energy release rate function $g(\alpha)$ of LEFM, which was previously shown to provide the correct effect of specimens geometry on the size effect (of Type 2) in specimens with deep notches or cracks, does not give a correct transition to the size effect (of Type 1) in specimens with no notch or crack. The reason is that the energy release rate of an initiating crack vanishes, which forces the use of higher-order derivatives of $g(\alpha)$. However, differentiation of a functional approximation is known to always greatly increase the approximation error.

2. To remedy the problem, the effective structure size for the Type 1 size effect is now characterized in terms of the ratio of the strain at surface to the gradient of that strain. In theory, this 
ratio should apply regardless of the structure geometry, but experimental data to check this are unavailable.

3. Fracture parameters $G_{f}$ and $c_{f}$ must be identified separately by fitting only the size effect data from deeply notched specimens $\left(\alpha=0.3\right.$ and 0.15 ), and parameters $f_{r \infty}, D_{b}, l_{p}$, and $r$ separately by fitting only the size effect data for unnotched specimens $(\alpha=0)$. The parameters of the transition function $k, p$, and $q$ are then identified by fitting the entire data set.

4. The new USEL fits the measured nominal strength quite well, with an overall COV of only $2.3 \%$.

5. The new USEL also fits well the individual crack length and size effect data, with the two largest COVs equal to 3.8 and $3.2 \%$ and the rest of less than $3 \%$.

\section{Acknowledgments}

Financial support from the U.S. DOT, provided through Grant No. 227740 from the Infrastructure Technology Institute of Northwestern University, is gratefully appreciated. Further support for theoretical study was provided by the U.S. National Science Foundation under Grant No. CMMI-1129449 to Northwestern University.

\section{References}

Bažant, Z. P. (1984). "Size effect in blunt fracture: Concrete, rock, metal." J. Eng. Mech., 10.1061/(ASCE)0733-9399(1984)110:4(518), 518-535.

Bažant, Z. P. (1995). "Scaling of quasibrittle fracture: I. Asymptotic analysis based on laws of thermodynamics. ii. The fractal hypothesis, its critique and weibull connection.” Bulletin 22 95-7/C402s, Dept. of Civil Engineering, Northwestern Univ., Evanston, IL.

Bažant, Z. P. (1996). "Size effect aspects of measurement of fracture characteristics of quasibrittle material." Adv. Cem. Base. Mater., 4(3/4), 128-137.

Bažant, Z. P. (1997). "Scaling of quasibrittle fracture: Asymptotic analysis." Int. J. Fract., 83(1), 19-40.

Bažant, Z. P. (2005). Scaling of structural strength, Elsevier ButterworthHeinemann, Burlington, MA.

Bažant, Z. P., and Becq-Giraudon, E. (2002). "Statistical prediction of fracture parameters of concrete and implications for choice of testing standard." Cem. Concr. Res., 32(4), 529-556.

Bažant, Z. P., and Kazemi, M. T. (1991). "Size dependence of concrete fracture energy determined by rilem work-of-fracture method." Int. J. Fract., 51(1), 121-138.

Bažant, Z. P., Kazemi, M. T., and Gettu, R. (1989). "Recent studies of size effect in concrete structures," A. H. Hadjian, ed., Proc., Transactions, 10th Int. Conf. on Structural Mechanics in Reactor Technology, Vol. H, Los Angeles, 85-93.

Bažant, Z. P., and Li, Z. (1995). "Modulus of rupture: Size effect due to fracture initiation in boundary layer." J. Struct. Eng., 10.1061/(ASCE) 0733-9445(1995)121:4(739), 739-746.

Bažant, Z. P., and Li, Z. (1996). "Zero-brittleness size-effect method for one-size fracture test of concrete." J. Eng. Mech., 10.1061/(ASCE) 0733-9399(1996)122:5(458), 458-468.

Bažant, Z. P., and Pfeiffer, P. A. (1987). "Determination of fracture energy from size effect and brittleness number." ACI Mater. J., 84(6), 463-480.

Bažant, Z. P., and Planas, J. (1998). Fracture and size effect in concrete and other quasibrittle materials, CRC Press, Boca Raton, FL.

Bažant, Z. P., Vořechovský, M., and Novák, D. (2007). "Asymptotic prediction of energetic-statistical size effect from deterministic finite- element solutions." J. Eng. Mech., 10.1061/(ASCE)0733-9399(2007) 133:2(153), 153-162.

Bažant, Z. P., and Yu, Q. (2004). "Size effect in concrete specimens and structures: New problems and progress," Proc., 5th Int. Conf. on Fracture Mechanics of Concrete and Concrete Structures, V. C. Li, K. Y. Leung, K. J. Willam, and S. L. Billington, eds., Vol. 1, Balkema, Rotterdam, Netherlands, 153-162.

Bažant, Z. P., and Yu, Q. (2009). "Universal size effect law and effect of crack depth on quasi-brittle structure strength." J. Eng. Mech., 10.1061/ (ASCE)0733-9399(2009)135:2(78), 78-84.

Bažant, Z. P., and Yu, Q. (2011). "Size-effect testing of cohesive fracture parameters and nonuniqueness of work-of-fracture method." J. Eng. Mech., 10.1061/(ASCE)EM.1943-7889.0000254, 580-588.

Becq-Giraudon, E. F. (2000). "Size effect on fracture and ductility of concrete and fiber composites." Ph.D. thesis, Northwestern Univ., Evanston, IL.

Carpinteri, A., Chiaia, B., and Ferro, G. (1995). Multifractal scaling law: An extensive application to nominal strength size effect of concrete structures, No. 50, Atti del Dipartimento di Ingegneria Strutturale, Politecnico de Torino, Italy.

Coleman, T. F., and Li, Y. (1994). "On the convergence of reflective Newton methods for large-scale nonlinear minimization subject to bounds." Math. Program, 67(1-3), 189-224.

Coleman, T. F., and Li, Y. (1996). "An interior, trust region approach for nonlinear minimization subject to bounds." SIAM J. Optim., 6(2), $418-445$.

Cusatis, G., and Schauffert, E. (2009). "Cohesive crack analysis of size effect." Eng. Fract. Mech., 76(14), 2163-2173.

Hoover, C. G., and Bažant, Z. P. (2013). "Comprehensive concrete fracture tests: Size effects of types 1 and 2, crack length effect and postpeak." Eng. Fract. Mech., 110, 281-289.

Hoover, C. G., and Bažant, Z. P. (2014). "Comparison of the Hu-Duan boundary effect model with the size-shape effect law for quasi-brittle fracture based on new comprehensive fracture tests." J. Eng. Mech., 10.1061/(ASCE)EM.1943-7889.0000632, 480-486.

Hoover, C. G., Bažant, Z. P., Vorel, J., Wendner, R., and Hubler, M. H. (2013). "Comprehensive concrete fracture tests: Description and results." Eng. Fract. Mech., 114, 92-103.

Irwin, G. R. (1958). "Fracture." Handbuch der physik, S. Flgge, ed., Springer, Berlin, 551-590.

Karihaloo, B. L., Abdalla, H. M., and Xiao, Q. Z. (2003). "Size effect in concrete beams." Eng. Fract. Mech., 70(7-8), 979-993.

Malvar, J. L., and Warren, G. E. (1988). "Fracture energy for threepoint-bend tests on single-edge-notched beams." Exp. Mech., 28(3), 266-272.

Nallathambi, P. (1986). "Fracture behaviour of plain concretes." Ph.D. thesis, Univ. of New Castle, Callaghan, Australia.

Petersson, P. E. (1981). "Crack growth and development of fracture zone in plain concrete and similar materials." Rep. No. tvbm-1006, Div. of Building Materials, Lund Institute of Technology, Lund, Sweden.

Rocco, C. G. (1995). "Size dependence and fracture mechanisms in the diagonal compression splitting test." Ph.D. thesis, Dept. Ciencia de Materiales, Universidad Politecnica de Madrid, Madrid, Spain.

Sabnis, G. M., and Mirza, S. M. (1979). "Size effect in model concretes." J. Struct. Div., 105(6), 1007-1020.

Tang, T., Bažant, Z. P., Yang, S., and Zollinger, D. (1996). "Variable-notch one-size test method for fracture energy and process zone length." Eng. Fract. Mech., 55(3), 383-404.

Timoshenko, S. P., and Goodier, J. N. (1951). Theory of elasticity, McGraw Hill, New York.

Weibull, W. (1939). "The phenomenon of rupture in solids." Proc. R. Swedish Inst. Eng. Res., 153, 1-55.

Weibull, W. (1951). "A statistical distribution function of wide applicability." J. Appl. Mech., 18, 293-297. 